\title{
A COMPACT AND BROADBAND 90-DEGREE WAVEGUIDE TWIST TRANSFORMER FOR INTEGRATED WAVEGUIDE SUBSYSTEMS
}

\author{
Uwe Rosenberg \\ Marconi Communications GmbH, D-71522 Backnang, Germany \\ Jens Bornemann \\ Dept. of Elec. \& Comp. Engr, University of Victoria, Victoria, BC, V8W 3P6 Canada \\ Smain Amari \\ Dept. of Elec. \& Comp. Engr, Royal Military College, Kingston, ON, K7K 7B4 Canada
}

\begin{abstract}
A novel 90-degree waveguide twist is presented. The design features compactness by utilizing a central triple-mode quarter-wave transformer region between input and output waveguides of different cross sections. Return-loss values in excess of $30 \mathrm{~dB}$ are obtained over an operating bandwidth close to 20 percent. Larger bandwidths are obtained by using additional quarter-wave steps preceding and/or succeeding the central transformer region. Several different design examples at 4,6, 19 and $26 \mathrm{GHz}$ are presented for this unique 90degree twist transformer approach. Experimental verification is provided in the 6 and $19 \mathrm{GHz}$ range.
\end{abstract}

\section{INTRODUCTION}

Among a wide variety of polarization rotating components, 90-degree rectangular waveguide twists play an important role for interconnections of waveguide systems or subsystems. Earlier, twisted waveguide structures have been experimentally designed, e.g., [1], [2]. With the advent of modern integrated waveguide technology, several restrictions are placed on the realization of waveguide twist sections. They must be computer-aided designed, fabricated without post-assembly tuning and realizable by CNC milling techniques as part of integrated waveguide structures. Moreover, they need to cover a reasonable bandwidth and must be as short as possible. Two attempts to satisfy such requirements have been reported. The continuously varying L-shaped design in [3] can readily be machined but is several wavelengths long and does not lend itself to fast CAD procedures. The design in [4] is built on a series of rectangular waveguide sections and satisfies most of the above expectations. Its major drawback, however, is the exceptional low bandwidth due to the utilization of only a single return-loss zero in the stepped junction between the input and output guides.

Therefore, this paper introduces a new design which is based on a central triple-mode quarterwave transformer region in order to enhance considerably the bandwidth of the twists in [4], while maintaining a compact structure and the possibility of impedance transformation; i.e., input and output waveguides can have different geometries. The principal layout is depicted in Fig. 1. The central transformer region is based on the propagating $\mathrm{TE}_{10}, \mathrm{TM}_{11}$, and $\mathrm{TE}_{01}$ triple-mode set representing electrically a three-section quarter-wave transformer. Hence, this design permits the creation of a relatively large operating bandwidth with excellent return-loss performance that can be further extended and improved by using additional transformer sections preceding and/or succeeding the central region. Several different computed and measured results demonstrate the validity of this unique design.

\section{THEORY}

The theoretical analysis of the compact twist is based on the coupled-integral-equations technique which combines several advantageous features, particularly for the computation of cascaded waveguide sections. (For details the reader is referred, e.g., to [5].) One of the salient 
features of the method is the simultaneous inclusion of all edge conditions. Let $a_{1}, b_{1}$ and $a_{2}$, $b_{2}$ in Fig. 2 be the cross-section dimensions of two connected waveguide sections at an arbitrary discontinuity in Fig. 1, then the basis function chosen to represent the electric field in the common aperture are

$$
\overrightarrow{\mathrm{X}_{\mathrm{ap}}}(\mathrm{x}, \mathrm{y})=\sum_{\mathrm{r}} \frac{\left[\nabla \mathrm{T}_{\mathrm{hr}}^{0} \times \overrightarrow{\mathrm{e}}_{\mathrm{z}}\right] \mathrm{c}_{\mathrm{r}}}{\left[\left(1-\frac{\mathrm{x}}{\mathrm{e}}\right)\left(\frac{\mathrm{x}}{\mathrm{g}}-1\right)\left(1-\frac{\mathrm{y}}{\mathrm{c}}\right)\left(\frac{\mathrm{y}}{\mathrm{d}}-1\right)\right]^{1 / 3}}+\sum_{\mathrm{s}} \frac{\left[-\nabla \mathrm{T}_{\mathrm{es}}^{0}\right] \mathrm{c}_{\mathrm{s}}}{\left[\left(1-\frac{\mathrm{x}}{\mathrm{e}}\right)\left(\frac{\mathrm{x}}{\mathrm{g}}-1\right)\left(1-\frac{\mathrm{y}}{\mathrm{c}}\right)\left(\frac{\mathrm{y}}{\mathrm{d}}-1\right)\right]^{1 / 3}}
$$

where $\mathrm{T}^{0}{ }_{\mathrm{hr}}$ and $\mathrm{T}^{0}$ es are the TE- and TM-mode cross-section functions of the common aperture, and $\mathrm{c}_{\mathrm{r}}$ and $\mathrm{c}_{\mathrm{s}}$ are the respective coefficients of the basis functions. If one or more walls of two connected guides are aligned, then the edge conditions of the respective mirror images are included.

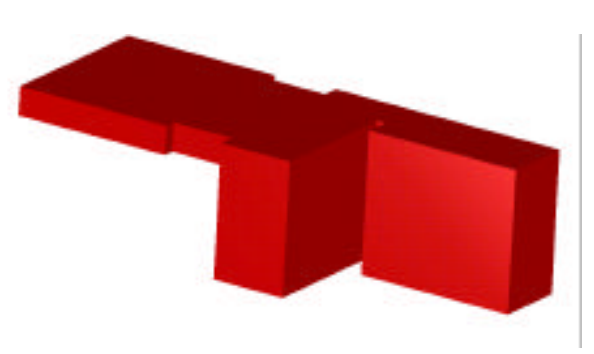

Fig. 1 Principal layout of compact and broadband 90-degree waveguide twist transformer.

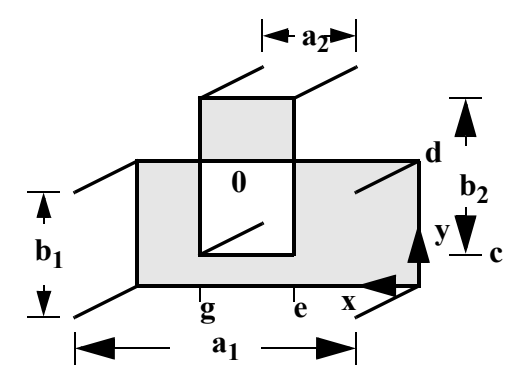

Fig. 2 Discontinuity formed by two offsetconnected rectangular waveguides.

The design of this novel twist initially starts with the rough dimensioning of the central transformer region having a square cross section and a length of approximately a quarter guided wavelength at the center frequency of the operating band. The interconnecting waveguides aligned at the lower (or upper) edge of the first cross section and at the right (or left) edge of the second one - are associated with irises or transformer sections for further refinement of the design. Final optimization of the complete structure as, e.g., in [6] fine tunes the design.

\section{RESULTS}

The twist design for WR137 waveguides serving the lower $6 \mathrm{GHz}$ band (5.8 to $6.4 \mathrm{GHz}$ ) shows extremely high performance (c.f. Fig. 3), i.e., the return-loss response exhibiting the characteristic three transformation zeros exceeds $40 \mathrm{~dB}$ over more than the 12 percent bandwidth. For experimental verification, this twist has been realized in two halves by CNC milling techniques (see photograph in Fig. 4). Despite the milling radii, the measured return loss exhibits excellent properties $(40 \mathrm{~dB})$ within the desired band and is in good agreement with the computed data.

Additionally, a $26 \mathrm{GHz}$ twist has been designed for a 20 percent frequency band considering an impedance transformation from a reduced-height input to a standard output waveguide (WR34) of aspect ratio 2:1. Over the desired frequency band, a return loss - with the inherent three zeros - of more than $30 \mathrm{~dB}$ (c.f. Fig. 5) is obtained which will suffice for the majority of applications.

In order to enhance the bandwidth, we use an additional quarter-wave transformer section preceding the central triple-mode region. The performance of a $4 \mathrm{GHz}$ design example for WR229 waveguides is shown in Fig. 6. The computed response demonstrates the excellent wide band performance based on the four return-loss zeros associated with the number of elec- 
trical transformer sections.

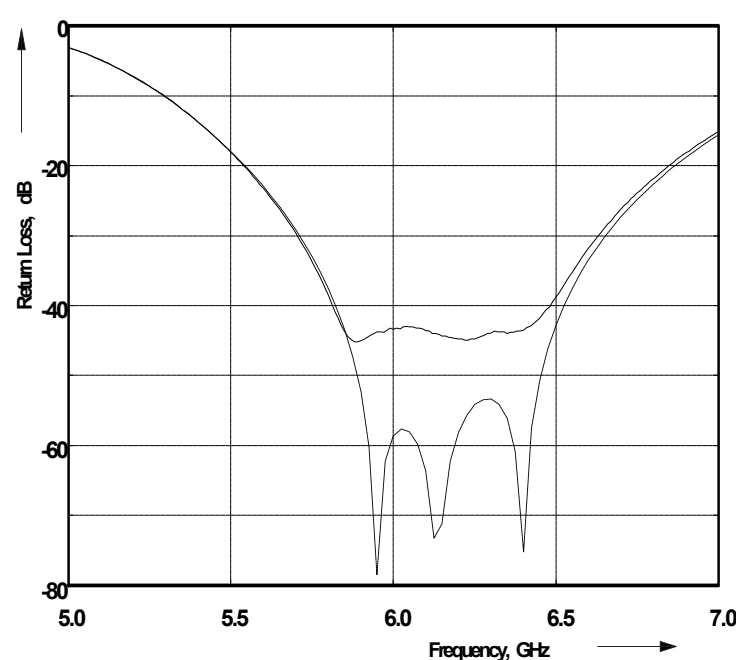

Fig. 3 Measured (light line) and computed (dark line) performance of $6 \mathrm{GHz}$ prototype twist transformer.

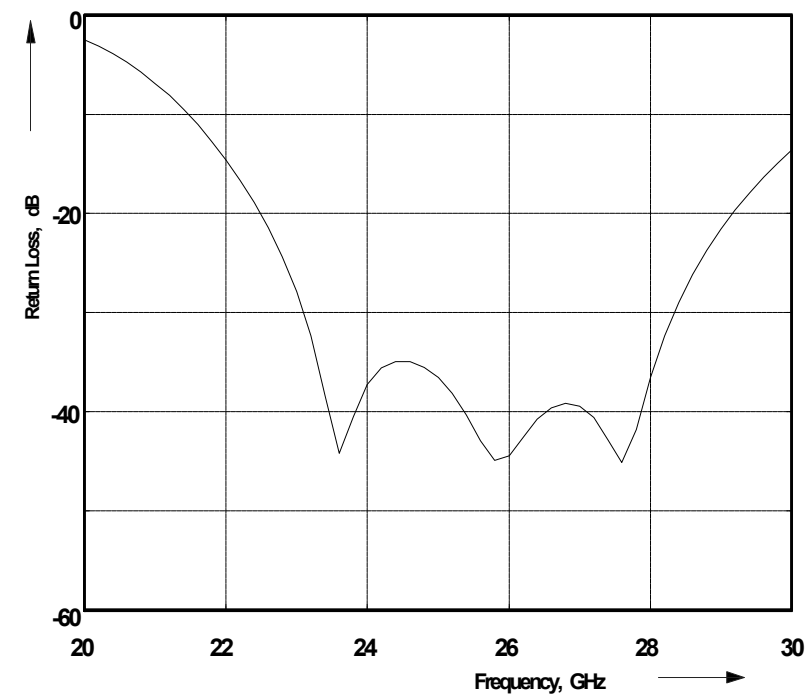

Fig. 5 Computed performance of triple-mode twist transformer in $26 \mathrm{GHz}$ band.

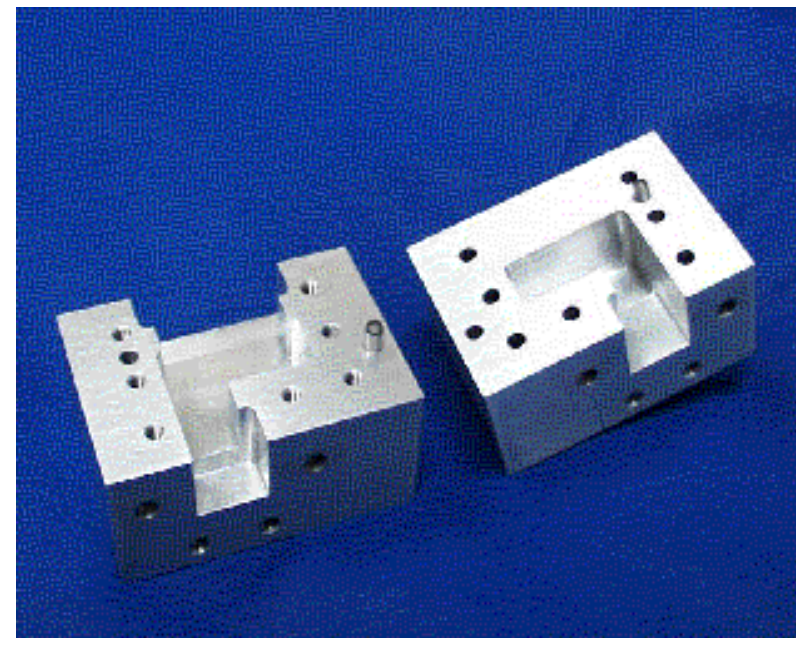

Fig. 4 Photograph of $6 \mathrm{GHz}$ prototype twist transformer.

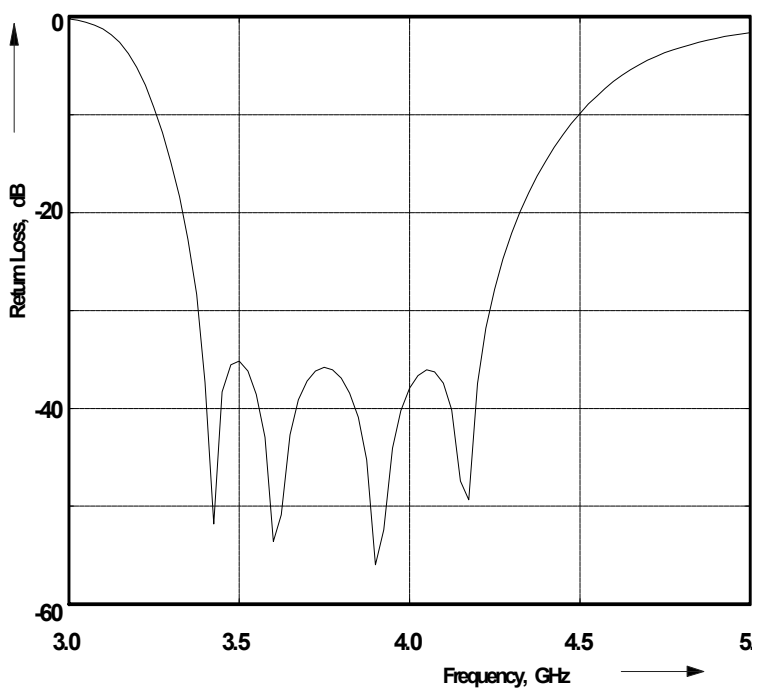

Fig. 6 Computed performance of quadruplesection twist transformer for the $4 \mathrm{GHz}$ band.

In modern integrated waveguide systems, milling radii need to be accounted for in order to avoid experimental fine adjustments [7]. Therefore, a regularly designed $19 \mathrm{GHz}$ twist including transformation from reduced-height to full-height waveguide (WR42) was redesigned for reasonable milling radii of $1.05 \mathrm{~mm}$; the length of the center section (incl. radii) is $4.5 \mathrm{~mm}$. A commercial software package providing the representation of the corner radii by boundary contour mode-matching (BCMM) library elements was used for this purpose [8]. Fig. 7 shows the comparison between measured and computed results. Between 17.5 and $20.6 \mathrm{GHz}$, the return loss is better than $35 \mathrm{~dB}$. The photograph in Fig. 8 depicts the prototype of this design with milling radii. Notice the different waveguide cross sections at the input (reduced height, upper port) and output (full height, lower port).

\section{CONCLUSION}

A compact 90-degree twist transformer providing high performance over broad operating frequency bands has been established. The design is favorably suited for integrated waveguide 
subsystems. It is based on a central triple-mode quarterwave transformer region that simultaneously permits impedance matching of different interface waveguide cross sections. Determination of the geometry with exact performance prediction is obtained by the application of effective EM CAD tools. Validation of the design strategy is provided by several different designs for microwave and millimeter-wave frequency bands. Comparisons between computed and measured results involving same and different input/output cross sections at $6 \mathrm{GHz}$ and $19 \mathrm{GHz}$ demonstrate the effectiveness of the components. Moreover, the incorporation of milling radii is accomplished.

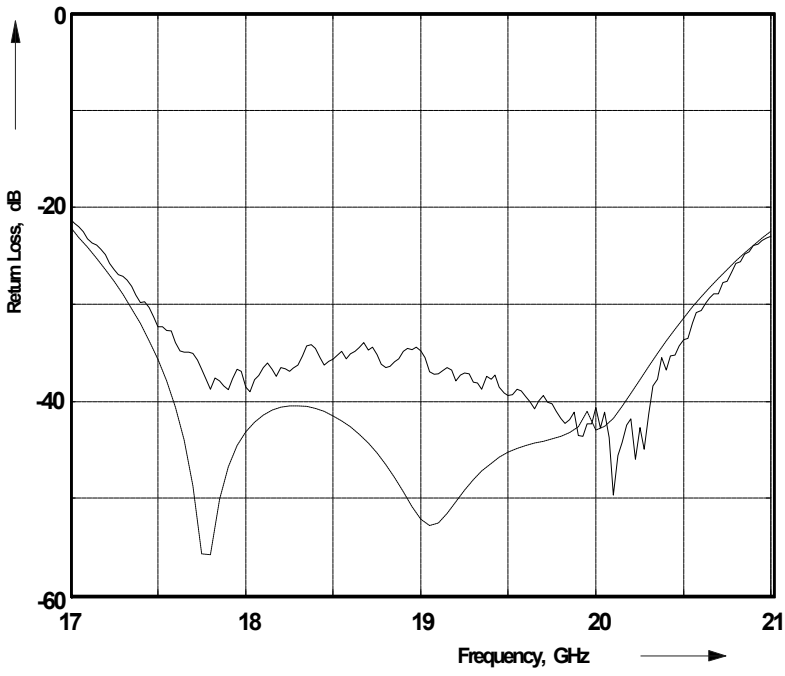

Fig. 7 Measured (light line) and computed (dark line) performance of $19 \mathrm{GHz}$ prototype twist transformer including milling radii of $1.05 \mathrm{~mm}$.

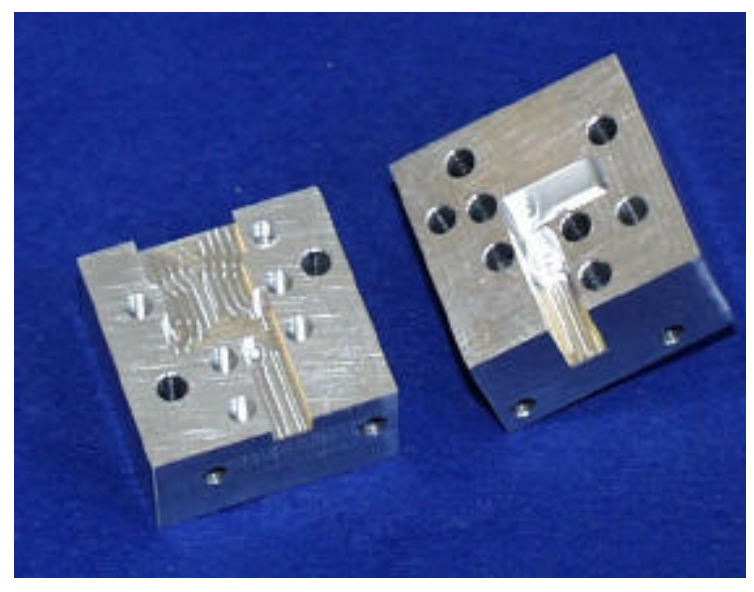

Fig. 8 Photograph of the $19 \mathrm{GHz}$ prototype twist transformer with milling radii. Note the transformation from reduced-height to full-height waveguide

\section{REFERENCES}

[1] H.A. Wheeler and H. Schwiebert, "Step-twist waveguide components", IRE Trans. Microwave Theory Tech., vol. MTT-3, pp. 44-52, Oct. 1955.

[2] B.C. DeLoach, "Step-twist-junction waveguide filters", IRE Trans. Microwave Theory Tech., vol. MTT-9, pp. 130-135, Mar 1961.

[3] H.F. Lenzing and M.J. Gans, "Machined waveguide twist", IEEE Trans. Microwave Theory Tech., vol. 38, pp. 942-944, July 1990.

[4] J. Bornemann, "Design of integrated waveguide twist components", IEE Proc.-Microw. Antennas Propag., Vol. 142, pp. 345-349, Aug. 1995.

[5] J. Bornemann, S. Amari and R. Vahldieck, "Analysis of waveguide discontinuities by the coupled-integral-equations technique", Recent Res. Devel. Microwave Therory \& Tech., Vol. 1, pp. 25-35, Dec. 1999.

[6] K. Madsen, H. Schaer-Jacobsen and J. Voldby, "Automated minimax design of networks,” IEEE Trans. Circuits Systems, Vol. CAS-22, pp. 791-796, Oct. 1975.

[7] R. Beyer, J. Bornemann, U. Rosenberg and J. Uher, "CAD of waveguide components for antenna feed systems: State-of-the-art", in Proc. 8th Int. Symp. Microwave Optical Technology, pp. 413-420, Montreal, Canada, June 2001.

[8] Mician, $\mu$ Wave Wizard 4.0, User's Manual, 2001 (www.mician.com). 